\title{
REVIEW
}

\section{Management of systemic risk factors for chronic tendinopathy}

\section{Gestion des facteurs de risque systémiques des tendinopathies chroniques}

\author{
C. Kabore $^{a}$, Q. Salier ${ }^{a}$, P. Geerts ${ }^{b}$, J.-F. Kaux ${ }^{c, *}$
}

\begin{abstract}
a Department of Orthopaedic Surgery, University and University Hospital of Liège, Avenue de l'Hôpital, B35, 4000 Liège, Belgium

b Department of Orthopaedic \& Hand Surgery, Centre Hospitalier Régional du Val de Sambre (CHRSV), Rue Chère Voie 75, 5060 Auvelais, Belgium

c Department of Physical Medicine and Sports Traumatology, SportS2, FIFA Medical Centre of Excellence, IOC Research Centre for Prevention of Injury and Protection of Athlete Health, FIMS collaborative Center of Sports Medicine, University and University Hospital of Liège, Liège, Belgium
\end{abstract}

Received 10 June 2020; accepted 26 December 2020

Available online 12 January 2021

\author{
KEYWORDS \\ Tendinopathy; \\ Chronic \\ tendinopathy; \\ Systemic risk factor
}

\begin{abstract}
Summary
News. - In the last twenty years, sports activities have become increasingly important in our modern society, and tendinopathy is a common overuse injury in the athletic and professionally active population. The etiology of tendon pathology, although still poorly understood, is multifactorial and involves both intrinsic and extrinsic risk factors, which could precipitate the occurrence of the pathology in predisposed individuals. There are significant interindividual variations in the development and evolution of tendinopathy, making them a contemporary challenge in clinical practice and justifying the need to recognize and correct any risk factor as much as possible. Given the complexity of the etiology of tendon pathology and the difficulty to establish their effective and long-term management, general risk factors must be searched and identified in any patient with chronic or recurrent tendinopathy.

Conclusion. - Through this review, we will focus on the various systemic risk factors highlighted in the context of chronic tendinopathy, before proposing to address the principles of precaution to deal with a patient subject to the pathology.

(c) 2021 Elsevier Masson SAS. All rights reserved.
\end{abstract}

\footnotetext{
* Corresponding author. Department of Physical Medicine and Sports Traumatology, SportS2, FIFA Medical Centre of Excellence, IOC Research Centre for Prevention of Injury and Protection of Athlete Health, FIMS collaborative Center of Sports Medicine, University and University Hospital of Liège, Liège, Belgium.

E-mail address: jfkaux@chu.ulg.ac.be (J.-F. Kaux).
} 


\section{MOTS CLÉS}

Tendinopathie ;

Tendinopathie

chronique ;

Facteur de risque

systémique

\section{Résumé}

Actualités. - Au cours des vingt dernières années, les activités sportives ont pris une importance croissante dans notre société moderne, et la tendinopathie est une blessure de surmenage courante dans la population active sportive et professionnelle. L'étiologie de la pathologie tendineuse, bien qu'encore mal comprise, est multifactorielle et implique des facteurs de risque à la fois intrinsèques et extrinsèques, qui pourraient précipiter l'apparition de la pathologie chez des individus prédisposés. Il existe d'importantes variations interindividuelles dans le développement et l'évolution des tendinopathies, ce qui en fait un défi contemporain dans la pratique clinique et justifie la nécessité de reconnaître et de corriger tout facteur de risque autant que possible. Étant donné la complexité de l'étiologie de la pathologie tendineuse et la difficulté d'établir leur prise en charge efficace et à long terme, les facteurs de risque généraux doivent être recherchés et identifiés chez tout patient atteint de tendinopathie chronique ou récurrente.

Conclusions. - À travers cette revue, nous nous concentrerons sur les différents facteurs de risque systémiques mis en évidence dans le cadre d'une tendinopathie chronique, avant de proposer d'aborder les principes de précaution à prendre face à un patient sujet à la pathologie. (c) 2021 Elsevier Masson SAS. Tous droits réservés.

\section{Keypoints}

- The etiology of tendinopathies, although still poorly understood, is multifactorial and involves both intrinsic risk factors, including biomechanical or genetic abnormalities, and extrinsic risk factors, in particular the conditions under which the stress is exerted, which could precipitate the pathology in predisposed individuals.

- Given the complexity of the etiology of tendinopathies and the difficulty of effective and long-term management of these tendinopathies, the identification of general risk factors must be carried out in any patient with chronic or recurrent tendinopathies.

- Through this review, we will focus on the various general factors highlighted in the context of chronic tendinopathies, before proposing to address the principles of precaution and development to deal with a patient subject to the pathology.

\section{Introduction}

Chronic tendinopathies are frequent and represents disabling musculoskeletal disorders, well-known to the general and sports populations. They can persist for years, manifested by persistent pain with functional impairment, preventing participation in both leisure and professional activities [1].

In the last twenty years, sports activities have become increasingly important in our modern society, and tendinopathy is a common overuse injury in the athletic and working populations [2]. The etiology of tendon pathology, although still poorly understood, is multifactorial and involves both intrinsic and extrinsic risk factors, which could precipitate the pathology in predisposed individuals. Tendons are connective tissues that behave like a dynamic entity that restructures permanently. They are frequently subject to injury during participation in physical activities; however, tendinopathy can also occur without the notion of excessive effort, within the framework of intercurrent pathologies that must be taken into consideration in the overall care of the patient $[3,4]$.

There are significant interindividual variations in the development and evolution of tendinopathy, making them a contemporary challenge in clinical practice and justifying the need to recognize and correct any identified risk factor. In recent decades there has been an increase in the number of publications concerning risk factors involved in the onset and persistence of tendinopathies. Through this review, we will focus on the various general factors involved in the onset of chronic tendinopathies, before proposing some principles of identification and prevention to apply to a patient subject to the pathology.

\section{Materials and methods}

\subsection{Source of studies and search strategy}

With our research, we wanted to determine the different general risk factors for chronic tendinopathies. We performed a bibliographic search on the Medline database via PubMed, Scopus and the Cochrane Central Registry of Ovid Controlled Trials, using different combinations of the words « tendon », « tenocyte », « tendinopathy », « chronic », « intrinsic », « extrinsic » and « risk factor ». The selected studies, published between 1987 and 2019, were heterogeneous regarding the benefits or adverse effects on the risk of tendinopathies, whether in humans or animals.

\subsection{Study selection and eligibility criteria}

Either original and review articles were selected, and titles and abstracts were reviewed. The full text was retrieved and reviewed if the abstract suggested that this might be a study in our topic of interest. Article reference lists from general reviews, systematic reviews, and 
Table 1 Classification of general risk factors for tendinopathy (non-exhaustive list).

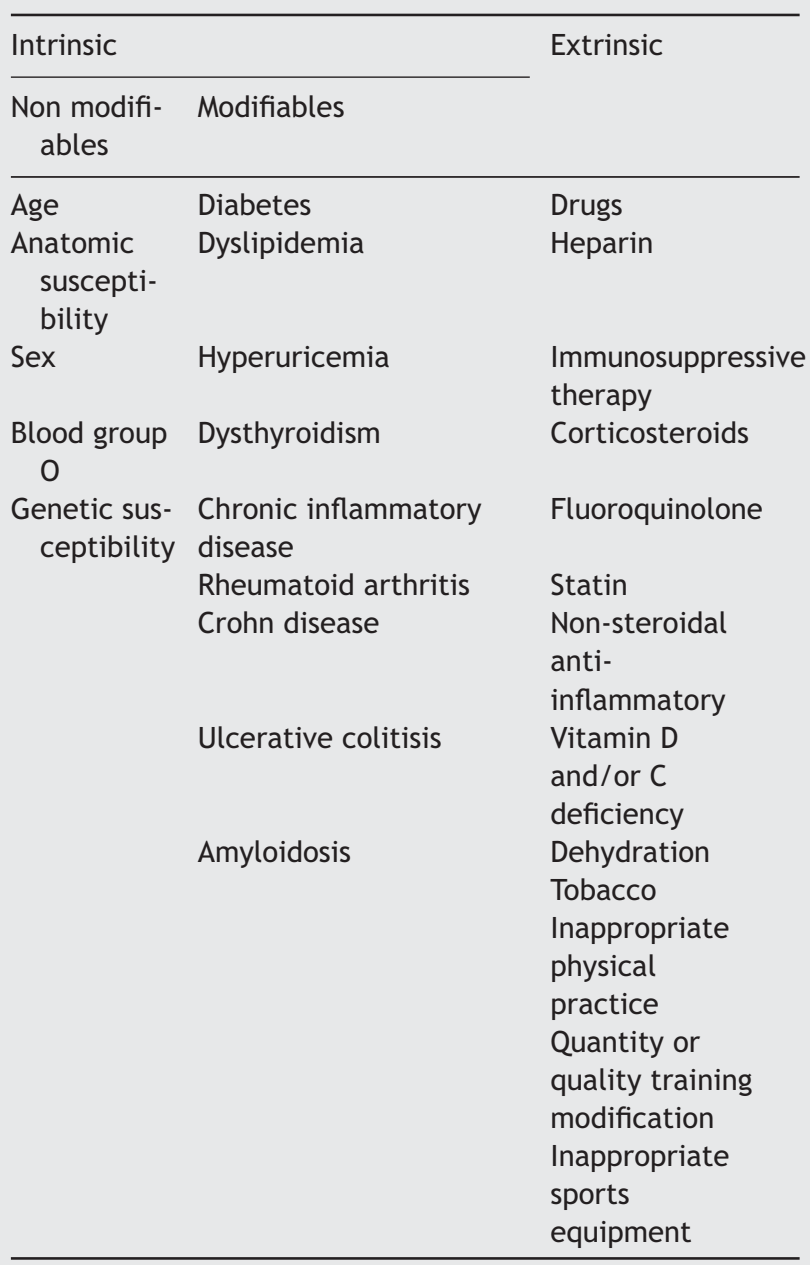

meta-analyses obtained from the search were examined in order to find any other original research investigation. Publications in English, French and German were included. Finally, in order to limit our review to the general and systemic risk factors of tendinopathy, we did not take in consideration specific anatomical risk factors concerning a localized tendinopathy (for example the anatomical features of the acromion in rotator cuff tendinopathies), which constitute for each localization an entire subject of research.

\subsection{Results}

After database search, we have obtained various general risk factors that we have classified between extrinsic and intrinsic risk factors, the latter being divided into modifiable and non modifiable intrinsic risk factors, as shown in Table 1. At the end of our review, recommendations for the management of general risk factors for tendinopathy are proposed in regard to either a general or a sport population of patients, as well as an example of a clinical management in Table 2.
Table 2 Tendinopathy: an exemple of clinical management.

\begin{tabular}{|c|c|}
\hline \multicolumn{2}{|l|}{ ANAMNESIS } \\
\hline \multicolumn{2}{|l|}{ Age } \\
\hline \multicolumn{2}{|c|}{ Family or personnal history of tendinopathies? } \\
\hline \multicolumn{2}{|c|}{ Diabetes, dysthyroidism or chronic inflammatory disease? } \\
\hline \multicolumn{2}{|c|}{ Blood group? } \\
\hline \multicolumn{2}{|c|}{ Last biology: } \\
\hline \multicolumn{2}{|c|}{ Hypercholesterolism, hyperuricemia or vitamin } \\
\hline \multicolumn{2}{|c|}{ deficiency? } \\
\hline \multicolumn{2}{|c|}{ Familial hypercholesterolemia? } \\
\hline \multicolumn{2}{|c|}{ Episode of gouty arthritis? } \\
\hline \multicolumn{2}{|c|}{ History of anatomic trouble } \\
\hline \multicolumn{2}{|c|}{ Medication: } \\
\hline \multicolumn{2}{|c|}{$\begin{array}{l}\text { Heparin, immunosuppressive therapy, corticosteroids, } \\
\text { antibiotics, statin, non-steroidal anti-inflammatory? }\end{array}$} \\
\hline \multicolumn{2}{|c|}{ Smoking? } \\
\hline \multicolumn{2}{|l|}{ Physical activity } \\
\hline \multicolumn{2}{|c|}{ Quantity or quality training modification } \\
\hline \multicolumn{2}{|c|}{ Inappropriate sports equipment } \\
\hline \multicolumn{2}{|c|}{ CLINIC } \\
\hline \multicolumn{2}{|c|}{ Tendinous palpation pain } \\
\hline \multicolumn{2}{|c|}{ Isometric contraction pain or isotonic excentric and } \\
\hline \multicolumn{2}{|c|}{ concentric against resistance pain } \\
\hline \multicolumn{2}{|c|}{ Tendinous passive stretching pain } \\
\hline PARACLINIC EXAMS & \\
\hline BLOOD ANALYSIS & \\
\hline Blood cell count & \\
\hline Red cells & \\
\hline Hematocritis & \\
\hline White cells & \\
\hline Blood group $A B O-$ Rhes & \\
\hline Creatinin & \\
\hline GFR & \\
\hline Uric acid & \\
\hline Sodium & \\
\hline Vitamin D & \\
\hline CRP & \\
\hline VS & \\
\hline Blood glucose & \\
\hline $\mathrm{HbA1c}$ & \\
\hline Triglycerid & \\
\hline Total cholesterol & \\
\hline LDL-cholestérol & \\
\hline HDL-cholestérol & \\
\hline TSH & \\
\hline Free T4 & \\
\hline Estradiol & \\
\hline Testosterone & \\
\hline IMAGING & \\
\hline ECHOGRAPHY & Calcifications? \\
\hline & Fibers discontinuity? \\
\hline & Peri- and/or \\
\hline & intratendinous oedema? \\
\hline NUCLEAR MAGNETIC & Asymptomatic \\
\hline RESONANCE & tendinopathy discovery? \\
\hline
\end{tabular}




\section{Systemic risk factors}

\subsection{Non modifiable intrinsic risk factors}

\subsubsection{Ageing}

Natural ageing during life is responsible for a chronic degeneration of tendons and a progressive dysfunction of the tendinous homeostasis. It has been demonstrated that there is a decrease in blood flow in the regions involved, as well as a progressive alteration of the metabolism of tenocytes. Several studies have shown the factors of the tendons natural ageing process, including inflammageing, reactive oxygen species formation, ageing stem cell population and glycation of matrix proteins [5-7].

Moreover, the tendons stiffness increases with age, resulting in the decrease of the capacity of the tendon to tolerate the repetition of a mechanical stress $[8,9]$.

In practice, a recent systematic review of rotator cuff diseases has identified a high rate of rotator cuff abnormalities in both symptomatic and asymptomatic patients, increasing with age to a rate high enough for degeneration of the rotator cuff to be considered a common aspect of normal human ageing [10].

\subsubsection{Gender}

Gender has been shown to be linked with tendinopathy by the circulating levels of sex hormones. Estrogen receptors have been identified in tendon tissue, and a study demonstrated a positive correlation between estradiol's blood levels and collagen synthesis rate [11]. For instance, women have lower limb tendinopathy during pre-menopausal years; the risk of tendon pathology and rupture become similar between men and women after menopause [12]. They have a higher proportion of type III collagen which confers a greater tendinous laxity [13].

However, it has been shown that the rate of collagen synthesis is lower in women than in men, which may contribute to a lower rate of tissue repair after exercise [14]: a comparative study has shown that weight-normalized patellar tendon \& Achilles tendon cross-sectional areas and stiffness were similar in trained and untrained female runners, while for males the cross sectional area increases with training: collectively, these findings indirectly suggest that the ability of Achilles and patellar tendons to adapt in response to habitual loading such as running is attenuated in women [15].

Pathological steroidal sex hormones rate therefore could be involved in the pathogenesis of tendon pathology. A study suggested that hormone replacement therapy taken by active post-menopausal women may operate by reducing the incidence of tendon abnormality and significantly less tendon thickness, but other studies are needed to evaluate the real impact of hormonal imbalance on tendinopathy in order to include them definitively as risk factors [16].

\subsubsection{Blood group}

It appears that patients in group 0 could be more susceptible to tendon ruptures than the other groups and that patients in group A could be the least likely to develop them [17]. At the time, this study corroborated with the results of another study conducted by Witschew et al., who had demonstrated the existence of a protein involved in tendinous homeostasis, $\mathrm{N}$-acetylgalactosamine transferase [18]. They were able to prove that its activity was higher in individuals in group A (less affected by tendon ruptures) while it was lower in individuals in group 0 (most affected by tendon ruptures). It was illustrated through a study among 832 patients with a tendon rupture, the frequency of blood group 0 being significantly higher $(52.8 \%)$, and, among the 83 cases of multiple ruptures or re-rupture, $68.7 \%$ of the patients having a group O blood [17-19].

However, it has to be noticed that in a more recent study, no correlation was found between blood group and the probability to develop achilles tendon rupture, this study being nevertheless on fewer individuals [20].

\subsubsection{Genetic factors}

Concerning tendinopathy, a study in twin patients revealed a heritability close to $40 \%$ for epicondylitis [21]. At the genetic level, any alteration affecting genes coding for the components of the tendons, such as collagen and the extracellular matrix, can lead to connective tissue diseases. Several studies carried out to find genetic factors involved in the onset of tendinopathy revealed the polygenic nature of the pathology and identified four genes whose alteration contributes to the onset of tendon pathology:

- the LOX gene codes for lysyl oxidase, an enzyme that facilitates the association of collagen and elastin fibers in the connective tissue;

- the ELN gene codes for elastin, which accounts for $2 \%$ of the dry weight of the tendons;

- the ITGB3 gene codes for glycoprotein IIla, which is a subunit of many integrins and receptor complexes in the extracellular matrix of tendons;

- the COL11A2 gene, coding for the alpha-2 strand of type $\mathrm{XI}$ collagen, which is particularly important in tendinous development.

These genes, previously identified as being involved in connective tissue diseases, are expressed abnormally in the context of tendinopathy, contributing to the functional characteristics of the pathology [22].

\subsection{Modifiable intrinsic risk factors}

\subsubsection{Diabetes mellitus}

Among the intercurrent pathologies associated with chronic tendinopathy, diabetes mellitus represents an important risk factor and a poor prognosis factor of the tendon pathology. It is also a risk factor for other musculoskeletal diseases, such as osteoporosis and joint stiffness [23].

From an epidemiological point of view, shoulder (mainly rotator cuff) and Achilles tendon injuries were more commonly observed in diabetic patients. Concerning the shoulder, both the retractile capsulitis and the cuff tendinopathy are more prevalent in diabetic patients compared to non-diabetic patients. Concerning the Achilles tendon, diabetes mellitus induces notable structural, inflammatory, and vascular changes in tendons, which may predispose diabetic patients to a greater risk of chronic tendinopathy and/or traumatic rupture [24]. 
From a morphological point of view, the pathological alterations observed in the tendons of diabetic patients consist in the loss of the white and glossy aspect, as well as a thickening of the tendon. Electron microscopy showed loss of collagen fibers' organization, decreased size and focal degeneration of the fibers without inflammatory cell infiltration. The number of elastin fibers was also reduced [25].

From a pathogenic point of view, several non-mutually exclusive physiopathological mechanisms explaining the occurrence of tendinopathies is recognized [24]. One of the well-studied phenomena is the excessive production of terminal glycation products (TGPs), which appear to play a significant role in the pathogenesis of diabetesrelated tendinopathies [26]. TGPs are glycated proteins or lipids which are non-enzymatically irreversibly oxidized after exposure to carbohydrates, which are increased in the diabetic patient. In human beings, it is generally the lysine side chains which are glycated, which are found abundantly in particular at the level of collagen [27].

\subsubsection{Dyslipidemia}

TGPs can also be formed by glycation of high and low-density lipoproteins responsible for the transport of cholesterol (respectively abbreviated HDL-C and LDL-C). The same mechanism seems to be associated with the tendinopathies of the Achilles tendon. Another theory explains that a high blood lipid concentration leads to the passage of LDL in the interstitial medium, thus creating an inflammatory reaction and producing, accordingly, their oxidation. These ox-LDLs recruit monocytes from the bloodstream and block them in the tendon. The macrophages will then phagocyte these ox-LDLs, thus causing the accumulation of foam cells, component of tendon xanthoma [28]. Logically, the patients with familiar hypercholesterolemia are predisposed to developed lipid-related tendinopathy, that may be expressed during the third decade of life through tendinous pain, and may be associated with the development of tendon xanthomas. However, associations between hypercholesterolemia and tendinopathy have also been identified in patients without familial dyslipidemia [24,29].

\subsubsection{Hyperuricemia}

Uric acid and intrarticular deposition of monosodium urate (MSU), well-known by rheumatologists to cause gout, may also be related to the occurrence of tendinopathies. Indeed, it has been shown that the deposition of intratendinous MSU induce an inflammatory reaction, apoptosis of the tendinocytes and consequently an alteration of the extracellular matrix. Together, these processes may lead to impaired tendon function, and limit the ability of the tendon to repair itself once damaged [30]. Recent progresses in imaging have allowed ultrasonic detection of the deposition of MSU crystals in tendinous structures. Particularly Achilles tendon, patellar tendon, triceps and quadriceps tendons are often affected [31]. Corroborating these data, Achilles tendon ruptures occur more often in asymptomatic patients with hyperuricemia than in asymptomatic normouricaemic subjects [32].

\subsubsection{Thyroids Hormones}

Thyroid hormones play a central role in regulating human metabolism. In regard to supportive tissue, a recent study on tenocytes has demonstrated the presence of thyroid hormone receptors on their surface. Activation of these receptors causes an anti-apoptotic effect and stimulates the tenocytes to produce extracellular matrix and, hence, collagen [33]. We know that hypothyroidism, an extremely common condition in women, causes the accumulation of glycosaminoglycans in the extracellular matrix, increasing the risk of calcification of the tendon, thus predisposing it to injury [34]. At last, it has been demonstrated that patients with simultaneously a tendinopathy and an endocrine pathology, for example a thyroid pathology, have early symptomatology, a longer natural history and undergo surgery more frequently, compared to a group of control individuals [35].

\subsubsection{Inflammatory pathologies}

It is estimated that about $2 \%$ of the occurrence of Achilles tendinopathies can be attributed to inflammatory joint disorders, such as rheumatoid arthritis [36]. A causal link has recently been demonstrated between the occurrence of periodontal disease and tendinopathies [37,38]. Another study has focused on the association between poor dental hygiene associated to gingivitis or periodontitis and the recurrence of injury in high-level football players, and the conclusions goes in the same way. The results of this preliminary study indicates that poor oral health is associated with repeated exercise-associated muscle cramps, muscle or tendon reinjury and multiple types of reinjury [39]. Specifically, in the case of onset of insertional tendinopathies (or enthesopathies), etiologies of chronic inflammatory diseases such as chronic inflammatory arthropathies, periodontal disease or chronic inflammatory bowel disease should be suspected [40].

From a pathogenic point of view, the important role of proinflammatory cytokines, such as tumor necrosis factor alpha (TNF $\alpha$ ) and interleukin 6 (IL-6) [41], is recognized in the pathogenesis of chronic tendinopathies despite the absence of inflammatory cells in or around the lesion [37]. This persistent systemic inflammation, even subclinical and of low grade, is thus responsible for pain and morphological alterations observed in tendinopathies. In addition to the consequences of the increasing inflammation on tendons, chronically higher levels of IL-6 and other cytokines are associated with fatigue, a serious risk factor for (re)injuries [42].

Another mechanism explaining the occurrence of tendinopathies in the context of chronic inflammatory diseases is the imbalance between matrix metalloproteinases (MMP) and their physiological inhibitors, TIMP (tissue inhibitors of metalloproteinases), leading to matrix destruction, worsening the repercussions of mechanical overload, and ultimately leading to a predisposition to tendon ruptures $[24,43]$.

It should also be noted, in the panel of intercurrent inflammatory pathologies, that several cases of amyloidosis have described spontaneous tendon ruptures and tenosynovites as first symptoms. The mechanism of action on tendon is not known but probably linked with to the effects (poten- 
tially inflammatory) of amyloid deposits in the tendon or synovial sheath [44].

\subsection{Extrinsic risk factors}

\subsubsection{Side effects of pharmacotherapy}

3.3.1.1. Corticosteroids. Some medicines should be avoided especially in active sportsmen and/or chronic tendinopathies. The use of corticosteroids, although still widespread in sports medicine, remains controversial on its safety at the tendinous level. Indeed, their use as injected therapy seems to bring a short-term benefit, in particular by reducing pain, but the occurrence of adverse effects is observed in nearly $82 \%$ of the therapeutic trials, including a decrease in tendon strength, atrophy or even tendon rupture [45]. These effects are due, on the one hand, to the alteration of the metabolism of the tenocytes and, on the other hand, to the vasoconstriction generated by activation of adrenergic receptors and inhibition of nitric oxide synthase (NOS) [46]. Therefore evidence remains too low to promote the injection of glucocorticoids in the context of tendinopathies.

3.3.1.2. Low molecular weight heparin. Thrombin is an enzyme known for its ability to activate platelets and to convert soluble fibrinogen to insoluble fibrin. Thrombin though has many biological properties similar to those of growth factors: it activates or releases matrix-bound mitogenic factors by catalysis, and can activate cell surface receptors [47].

Low molecular weight heparin (LMWH) inhibits the activity and the generation of thrombin by inhibiting coagulation factor Xa. It therefore has been considered that LMWH at a thromboprophylactic dose might inhibit tendon repair [48]. In a study concerning 86 rats and the controlled healing of their Achilles tendon, it has been shown that LMWH delays tendon repair if given continuously with an osmotic mini pump for seven days, causing a reduction in force at failure, a reduction of stiffness and a reduction in energy uptake; however if injected twice daily, LMWH had no effect on tendon healing, probably because the anti-factor $\mathrm{Xa}$ activity between injections returns to normal, allowing sufficient thrombin stimulation for repair. Those findings do not have been studied concerning human tendon; moreover they indicated the need for further investigation on impact of long-acting direct factors $\mathrm{Xa}$ and thrombin inhibitors in tendinopathy [49].

\subsubsection{Antibiotics. Fluroroquinolone-induced}

tendinopathy have been widely described in the scientific literature [50]. The severity of the tendon lesions seems to correlate with the conformation of the active molecule, in particular the substitution at the R-7 position in the quinolone core. Thus, there is a higher toxicity of levofloxacin and fleroxacin compared to ciprofloxacin and norfloxacin, the latter two not having this structural characteristic. The dose-dependent character of this side effect was also demonstrated. Risk factors for fluoroquinolone-induced tendinopathies include age over 60 , concomitant administration of corticosteroids and a history of solid organ transplantation. The role of oxidative stress, in particular by altering mitochondrial activity, seems to play an important role in the tendon toxicity of fluoroquinolones. It is also important to know that the molecule becomes photo-actived under exposure of natural or artificial utlraviolet light, resulting in the formation and accumulation of free radicals altering the mitochondrial functions [51].

An other type of antibiotics has been studied for its interaction with tendon repair. The tetracycline family of antibiotics has been shown to inhibit mammalian matrix metalloproteinases (MMPs) by a mechanism that is independent of their antimicrobial activity. Doxycycline is considered to be the most potent tetracycline MMP inhibitor, whose activity is mediated both by direct and indirect mechanisms of action [52]. The use of doxycycline has been explored in vivo and in vitro, with contradictory findings regarding its efficacy on tendon repair [53-55]. A study demonstrated the benefit of extended administration in rats with injured Achilles tendons, with improved collagen fibril organization and enhanced biomechanical properties (stiffness, ultimate tensile strength, maximum load to failure, and elastic toughness), compared with limited doxycycline administration up to 2 weeks after an injury and repair, the duration of doxycycline administration being an important factor in the resultant quality of tendon repair in part because of the variation of MMP activity after an injury [56]. This study, while suggesting that MMP inhibitors may improve the quality of repair after Achilles tendon ruptures, highlights the difficulty in achieving optimal timing of delivery and efficacy of improved tendon repair with MMP inhibitors. Further investigations are needed regarding the effects of MMP inhibitors on human tendons, on different types of repair model examined and on long-term consequences on the repaired tendon.

3.3.1.4. Statins. Tendinopathies induced by the administration of statins have been described, but the incidence is very low in view of the very high number of prescriptions in the population [57]. In a series of 96 cases of tendinous complications, tendinopathies were mostly Achilles tendinopathies (52\% of the cases) and occurred mainly during the first four months following initiation of treatment. Pathophysiological mechanisms have been proposed to explain the occurrence of tendinopathies during statin therapy, such as the secretion of MMPs that has been described in lung fibroblasts and endothelial cells [58], but they remain poorly understood and hypothetical. Teams have, among other hypothesis, suggested the deleterious action of statins on the growth and differentiation of tenocytes [59].

In one hand, experimental studies in rats has showed that statins as simvastatin, atorvastatin and rosuvastatin caused a deterioration of the biomechanical properties of the Achilles tendon, with histopathological modifications such as foci of dystrophic calcification [60]. On the other hand, simvastatin as a statin has also been reported to reduce fibrosis and protects against muscle weakness in an experimental model in rats with a full-thickness supraspinatus tear [61]. Therefore, statins may be playing a detrimental or a potentially beneficial role on tendon properties depending on the amount, the timing and the type of statin therapy in a particular patient [62].

3.3.1.5. Nonsteroidal anti-inflammatory drugs (NSAIDs). The influence of nonsteroidal anti-inflammatory drugs (NSAIDs) on tendon repair remains controversial. Inflammation slows down the cicatricial process following collagen 
degradation and cell death and therefore increases the functional deficit in already hypocellular and hypovascular structures; however, it has been demonstrated that it triggers paradoxically the primary stage of tendon healing by the released growth factors and increased local blood flow, among other via prostaglandins, mediators of inflammation and potent vasodilators. These prostaglandins, produced by the cyclooxygenase enzymes COX-1 and COX-2 from arachidonic acid, are involved in both the inflammatory processes and in the initial stages of tendon healing [63]. For this reason, the use of nonsteroidal anti-inflammatory drugs (NSAIDs), although commonly used in the management of musculoskeletal injuries, should be carefully considered or even avoided. Studies have reported that NSAIDs could either improve [64], degrade [65], or have no influence on tendon repair [66]. This contradiction could be explained by the opposite effects of prostaglandins at several stages of repair, the specificity of anti-inflammatory drugs for constitutive COX-1 or inducible COX-2, the modes of administration, the effects specific to each NSAID and the anatomical site involved [63].

It has been confirmed the early involvement of inflammatory mechanisms at the muscular level by the research of B.M. Lapointe et al., and the negative impact with delayed rehabilitation in rats treated with diclofenac [67]. These effects were particularly obvious when administration was continued after the acute phase of the inflammatory reaction, reflecting an impairment of skeletal muscle repair and adaptation. Those results were linked to a diminished concentration of macrophage subpopulations in the damaged site, leading to an incomplete removal of necrotic tissues. Despite the absence of needed evidence concerning tendon repair, it could therefore be hypothesized the same chronology in tendon repair, and we therefore suggest limiting the use of NSAIDs in the first moments following the acute trauma, to allow the beneficial involvement of inflammatory mechanisms in tendon repair.

\subsubsection{Tobacco consumption}

Tobacco consumption is considered as a well known risk factor by the medical world, but should it be considered as a risk factor for tendinopathies? Many previous studies have demonstrated the negative impact of nicotine on fracture healing. A randomized animal study which aim was precisely to evaluate the impact of nicotine on the healing of seventy-two supraspinous tendons of rats, sectioned at level of the humeral head, demonstrated that the inflammation persisted longer, and that cell proliferation and collagen production were less important in the tendons of rats exposed to nicotine [68]. They concluded that nicotine delayed the healing of the supraspinatus tendon at the humerus in these rats and thus played a role in the failure of rotator cuff repair. A case-control study published in 2010 identified tobacco, or rather nicotine, known as a potent vasoconstrictor that reduces tissue-level oxygen delivery, as a contributing factor to the pathology of rotator cuff [69]. Subsequently, a study published in 2012 investigated the impact of preoperative smoking habits on rotator cuff tears and report a correlation between smoking habits and tearing of the rotator cuff, more precisely the size of the tear. They observed a proportional relationship between the severity of the cuff tears and both the average daily number of cigarettes and the total number of cigarettes smoked during life [70].

However, all the studies do not go in the same direction. Indeed, a case control study conducted in the United Kingdom in 2014 focused on quantifying the relative contributions of various risk factors, both constitutional and environmental, to rotator cuff pathology. This study ultimately excluded the involvement of previous or current smoking history on the genesis of rotator cuff tendinopathies [71].

From a molecular point of view tobacco increases the rate of reactive oxygen species (ROS). Besides, it is also known that these ROS cause intracellular damage to DNA and protein synthesis leading to cell apoptosis. There has not been yet a clinical study on the effect of ROS on tenocytes, but these effects have been demonstrated in other sites rich in type 1 collagen, such as cutaneous tissue [72].

Most of these studies have been done concerning tendinopathies of the rotator cuff. Moreover, as we have seen, not all studies are going in the same direction on this subject. Further investigations must be carried out before concluding definitively on the link with these risk factors, for the rotator cuff and for other anatomical regions as well.

\subsubsection{Hydration}

The extracellular water content of a tendon declines from about $80 \%$ to $85 \%$ at birth to approximately $30 \%$ to $70 \%$ in old age. The decrease in water and mucopolysaccharide contributes to the age-dependent changes of stiffness of the tendon. This results in less organization of the collagen fibril, less stiffness and a reduction in the gliding properties [73].

Despite the general idea that dehydration is a risk factor of tendinopathy, it could be surprising to note that no correlation has been demonstrated between any level of hydration of a person and the risk of tendon injury, during a physical activity or not. A study has assessed the prevalence of trauma injuries in triathletes and has looked for contributing factors involved. The conclusion shows there was no significant association between prevalence of tendinopathy and hydration issues [74]. Once again, more group studies are needed to established a link between the level of hydration and the risk of tendinopathy, especially in any kind of physical activity.

\subsubsection{Nutrition}

There are still too few studies to conclude on a particular regimen that could prevent or manage tendinopathies. Moreover, most studies on the subject speak about the beneficial effects of certain nutrients on tendinopathies and do not speak much about the potential deleterious effects of others.

A recent systematic review analyzed the most commonly used nutraceuticals and their effects on tendons [75]. First, vitamin $C$, on the one hand has an antioxidant role and on the other hand acts as a co-factor for the hydroxylation of lysine and proline essential to collagen synthesis. Therefore, Vitamin C, which is mostly found in fruits and vegetables, could be a major contributor to prevention of tendinopathy and promotion of healing. 
Vitamin D, a vitamin that can be easily found in some fatty fish (herring, mackerel, salmon, tuna, cod liver oil...) and egg yolk, is known to have a direct impact on collagen synthesis by tendon fibroblasts. It is also essential to regulate inflammation-repair mechanisms in limiting MMP1 expression levels in skin-derived fibroblasts, reduce the induction of prostaglandine E2 (PGE2) in synovial fibroblasts and in ligament fibrobasts. Vitamin $D$ also inhibited osteobastic differentiation and calcification [76].

Curcumin seems to be an interesting spice in collagen damage as shown in some studies [78]. From a molecular point of view, it has been demonstrated on in vitro human tenocytes that the anti-inflammatory and anti-apoptotic action of curcumin was due to its action on the NF-kB signaling pathway. Other studies confirm its beneficial effect as an antioxidant and its ability to reduce advanced glication end product of collagen (AGE-collagen). Moreover, curcumin appears to inhibit angiogenesis and therefore may be useful in reducing hypervascularization found in inflammatory tendon [77]. However, those findings needed to be put in perspective of the low bioavailability of curcumin. This is linked to its rapid plasma clearance and to its conjugation in the intestinal lumen, reducing the water-soluble nature and therefore the resorption [78].

\section{Recommendations for the management of general risk factors}

In addition to the usual modalities of recommended management of tendinopathies at the present time (eccentric exercises, biophysical processes such as extracorporeal shockwaves and therapeutic ultrasound, injected therapies such as platelet-rich plasma...) $[2,40]$, the knowledge in a patient of general risk factors for tendinopathies should suggest an overall and continuous care. An example of a clinical management of general risk factors among either a general or a sports population is proposed in Table 2 .

In addition to medical control of intercurrent pathologies and regular control of the adequate sport equipment, caution should be exercised concerning intensive and prolonged practice of certain sports activities. A study of Fredberg et al. demonstrated a significant relationship between asymptomatic ultrasonographic abnormalities in the tendons, such as altered collagen fiber structure an mucoid degeneration, and the risk of developing symptoms [79]. The use of ultrasonography thus has been proposed, especially in soccer players, to diagnose tendon changes before they become symptomatic [80]. It is interesting to mention that, although neovascularization in the tendons increases in tendinopathy, there are conflicting results concerning the value of Doppler ultrasonography in tendinopathy management, and there is still a lack of consensus in registration and quantifying this neovascularization [81,82].

With regard to diabetes mellitus, strict control of the pathology, particularly of glucose level, is recommended to slow down the progression of symptomatic and asymptomatic tendinopathies, as well as to improve the recovery of the patient. Insulin administration demonstrated a decrease in TGP accumulation, reducing the induced alterations in diabetic tendons $[24,83]$.
Disease control, especially a controlled diet, and biological control are recommended for dyslipidemia. It also seems legitimate to seek tendinopathy during the interrogation and clinical examination of patients treated with statins, and in particular with patients having factors favoring the occurrence of this complication. Although tendinopathy does not jeopardize the benefit of the use of this treatment, whether in primary prevention or cardiovascular secondary prevention in patients, caution is recommended in case of prolonged physical exertion [59].

Concerning the hyperuricemia, a two-stage approach, firstly urate-lowering therapy designed to dissolve MSU crystals and, secondly keeping uric acid below saturation point for long-life has been recommended. Imaging can be used to evaluate outcomes until MSU crystals are dissolved [84].

Caution is advisable when a patient is to be covered by antibiotic therapy, in particular with fluoroquinolone. It is also important to inform the patient about the phototoxicity of the antibiotic, with the consequent increased harmfulness of sun exposure that may seem trivial [47]. Several studies have demonstrated an attenuation of the toxic effects of fluroquinolones on tenocytes during the concomitant administration of antioxidants such as $\mathrm{N}$ acetyl-cysteine, anethole dithiolethione or MitoQ, the latter being able to improve the mitochondrial membrane permeability $[85,86]$.

Finally, when the clinical history is unclear or the usual risk factors are not found, it should be kept in mind that tendinopathies may be the mode of entry for an underlying inflammatory pathology. A visit to the dentist can also be suggested to exclude an existing parodontal disease $[37,38]$.

At the pharmacological level, several molecules interacting with TGPs, both in terms of their formation (such as pyridoxamine and glucosamine) and their elimination (such as $\mathrm{N}$-phenacylthiazolium and soluble TGP receptor isoforms) are currently under study. These molecules have demonstrated, in experimental animal models, their efficacy in reducing diabetic tendinous complications due to AGE formation [87]. In addition, as previously stated, further investigations on human tendon are needed to incorporate the administration of doxycycline in regards of tendon repair.

The current cost of investigations at the genomic level and new methods of sequencing exomes or the complete genome do not currently allow their use in clinical routine in pathologies considered to be of low relative priority, including tendinopathies. Investigations of molecular and genomic interactions are nonetheless promising areas for further research [22].

\section{Conclusion}

Given the complexity of the etiology of tendinopathies and the difficulty of effective and long-term management of these tendinopathies, the identification of general risk factors must be carried out in any patient with chronic or recurrent tendinopathies, and particularly within the sport population. The objectives of this screening are on the one hand the optimization of the management, acting on the patient as a whole, and on the other hand the adoption of strategies of prevention of tendon pathologies in pre- 
disposed patients. Finally, the introduction of a medication known to promote the onset of tendinopathies must be made with the knowledge of the cause, both on the part of the clinician and the patient, allowing a lifestyle adaptation and an effective prevention of the tendinopathies induced during the duration of pharmacological treatment.

\section{Compliance with Ethical Standards}

Ethical approval: this article does not contain any studies with human participants or animals performed by any of the authors.

\section{Funding}

The authors declare that they do not have any financial disclosures.

\section{Disclosure of interest}

The authors declare that they have no competing interest.

\section{References}

[1] Ackermann PW, Renstrom P. Tendinopathy in sport. Sports Health 2012;2:193-201.

[2] Kaux JF, Forthomme B, Le Goff, et al. Current opinions on tendinopathy. J Sports Sci Med 2011;10:238-53.

[3] Kaux JF, Crielaard JM. Tendon et tendinopathie. J Traumatol Sport 2014;31:235-40.

[4] Meeuwisse WH. Assessing causation in sport injury: a multifactorial model. Clin J Sport Med 1994;4:166-70.

[5] Dakin SG, Dudhia J, Werling NJ, et al. Inflamm-aging and arachadonic acid metabolite differences with stage of tendon disease. PLoS One 2012, http://dx.doi.org/ 10.1371 /journal.pone.0048978.

[6] Kohler J, Popov C, Klotz B, et al. Uncovering the cellular and molecular changes in tendon stem/progenitor cells attributed to tendon aging and degeneration. Aging Cell 2013;12:988-99.

[7] Thorpe CT, Streeter I, Pinchbeck GL, et al. Aspartic acid racemization and collagen degradation markers reveal an accumulation of damage in tendon collagen that is enhanced with aging. J Biol Chem 2010;285:15674-81.

[8] Strocchi R, De Pasquale V, Guizzardi S, et al. Human Achilles tendon: morphological and morphometric variations as a function of age. Foot Ankle 1991;12:100-4.

[9] Tuite DJ, Renström PA, O'Brien M. The aging tendon. Scand J Med Sci Sports 1997;7:72-7.

[10] Teunis T, Lubberts B, Reilly BT, et al. A systematic review and pooled analysis of the prevalence of rotator cuff disease with increasing age. J Shoulder Elb Surg 2014;23:1913-21.

[11] Renstrom P, Ljungqvist A, Arendt E, et al. Non-contact $A C L$ injuries in female athletes: an International Olympic Committee current concepts statement. $\mathrm{Br} \mathrm{J}$ Sports Med 2008;42:394-412.

[12] Maffulli N, Waterston W, Squair J, et al. Changing incidence Achilles tendon rupture in Scotland: a 15 year study. Clin J Sports Med 1999;9:157-60.

[13] Sullivan BE, Caroll CC, Jemiolo B, et al. Effet of acute resistance exercise and sex on human patellar tendon structural and regulatory mRNA expression. J Appl Physiol 2009;106:468-75.
[14] Miller BF, Hansen M, Olesen JL, et al. Tendon collagen synthesis at rest and after exercise in women. J Appl Physiol 2006; 102:541-6.

[15] Westh E, Kongsgraad M, Bojsen-Moller J, et al. Effect of habitual exercise on the structural and mechanical properties of human tendon, in vivo, in men and women. Scand J Med Sci Sports 2008;18:23-30.

[16] Cook JL, Bass SL, Black JE. Hormone therapy associated with smaller Achilles tendon diameter in active postmenopausal women. Scand J Med Sci Sports 2007;17: $128-32$.

[17] Jozsa L, Balint JB, Kannus P, et al. Distribution of blood groups in patients with tendon rupture. An analysis of 832 cases. J Bone Joint Surg Br 1989;71:272-4.

[18] Witschew N, Tantschew P, Gertschew A. Beziechungen zwichen Blutgruppenzugehörigkeit und Knochenbruchheilung. Beitr Orthop Traumatol 1987;34:515-22.

[19] Kujala UM, Järvinen $M$, Natri $A$, et al. $A B O$ blood groups and musculoskeletal injuries. Injury 1992;23:131-3.

[20] Maffulli N, Reaper JA, Waterston SW, et al. ABO blood groups and achilles tendon rupture in the Grampian Region of Scotland. Clin J Sport Med 2000;10:269-71.

[21] Hakim AJ, Cherkas LF, Spector TD, et al. Genetic associations between frozen shoulder and tennis elbow: a female twin study. Rheumatology (Oxford) 2003;42: $739-42$.

[22] Saunders CJ, Jalali Sefid Dashti M, Gamieldien J. Semantic interrogation of a multi knowledge domain ontological model of tendinopathy identifies four strong candidate risk genes. Sci Rep 2016, http://dx.doi.org/10.1038/srep19820.

[23] Del Rosso A, Cerinic MM, De Giorgio, et al. Rheumatological manifestations in diabetes mellitus. Curr Diabetes Rev 2006;2:455-66.

[24] Lui PPY. Tendinopathy in diabetes mellitus patientsEpidemiology, pathogenesis, and management. Scand J Med Sci Sports 2017;27:776-87.

[25] Grant WP, Sullivan R, Sonenshine DE, et al. Electron microscopic investigation of the effects of diabetes mellitus on the Achilles tendon. J Foot Ankle Surg 1997;36:272-8.

[26] Goldin A, Beckman JA, Schmidt AM, et al. Advanced glycation end products: sparking the development of diabetic vascular injury. Circulation 2006;114:597-605.

[27] Ansari NA, Ali R. Glycated lysine residues: a marker for non-enzymatic protein glycation in age-related diseases. Dis Markers 2011;30:317-24.

[28] Sugiyama N, Marcovina S, Grown AM, et al. Immunohistochemical distribution of lipoprotein epitopes in xanthomas from patients with familial hypercholesterolemia. Am J Pathol 1992;141:99-106.

[29] Abate M, Schiavone C, Salini V, et al. Occurrence of tendon pathologies in metabolic disorders. Rheumatology 2013;52:599-608.

[30] Chhana A, Callon KE, Dray M, et al. Interactions between tenocytes and monosodium urate monohydrate crystals: implications for tendon involvement in gout. Ann Rheum Dis 2014;73:1737-41.

[31] Naredo E, Uson J, Jiménez-Palop M, et al. Ultrasound detected musculoskeletal urate crystal deposition: which joints and what findings should be assessed for diagnosing gout? Ann Rheum Dis 2014;73:1522-8.

[32] Pineda C, Amezcua-Guerra LM, Solano C, et al. Joint and tendon subclinical involvement suggestive of gouty arthritis in asymptomatic hyperuricemia: an ultrasound controlled study. Arthritis Res Ther 2011;13:R4.

[33] Oliva F, Berardi AC, Misiti S, et al. Thyroid hormones enhance growth and counteract apoptosis in human tenocytes isolated from rotator cuff tendons. Cell Death Dis 2013, http://dx.doi.org/10.1038/cddis.2013.229. 
[34] Oliva F, Misiti S, Maffulli N. Metabolic diseases and tendinopathies: the missing link. Muscles Ligaments Tendons J 2014;4:273-4.

[35] Harvie P, Pollards TC, Carr AJ. Calcific tendinitis: natural history and association with endocrine disorders. J Shoulder Elbow Surg 2007;16:169-73.

[36] Jarvinen TA, Kannus P, Paavola M, et al. Achilles tendon injuries. Curr Opin Rheumatol 2001;13:150-5.

[37] Kipgen L, Kaux JF, Rompen E, et al. Impact of oral hygiene on sport. Review of the literature. J Traumatol Sport 2015;32:41-5.

[38] Kipgen L, Kaux JF, Rompen E, et al. Relationship between oral hygiene and tendinopathy in sportsmen. Science Sports 2016;31:227-9.

[39] Solleveld H, Goedhart A, Vanden Bossche L. Associations between poor oral health and reinjuries in male elite soccer players: a cross-sectional self-report stud. BMC Sports Sci Med Rehabil 2015, http://dx.doi.org/10.1186/s13102-015-0004-y.

[40] Ackermann PW. Tendinopathy: understanding epidemiology, pathology, healing, and treatment. In: Gomes ME, Reis RL, Rodrigues MT, editors. Tendon regeneration. Boston: Academic Press; 2015. p. 113-47.

[41] Lago R, Gomez R, Otero M, et al. A new player in cartilage homeostasis: adiponectin induces nitric oxide synthase type II and pro-inflammatory cytokines in chondrocytes. Osteoarthritis Cartilage 2008;16:1101-9.

[42] Robson-Ansley PJ, de Milander L, Collins M, Noakes TD. Acute interleukin-6 administration impairs athletic performance in healthy, trained male runners. Can J Appl Physiol 2004;29:411-8.

[43] Tsai WC, Liang FC, Cheng JW, et al. High glucose concentration up-regulates the expression of matrix metalloproteinase-9 and -13 in tendon cells. BMC Musculoskelet Disord 2013;14:255.

[44] Benito JR, Martinez I, Monner J, et al. Primary amyloidosis presenting as extensor tenosynovitis. Plast Reconstr Surg 1999;103:556-8.

[45] Hart L. Corticosteroid and other injections in the management of tendinopathies: a review. Clin J Sport Med 2011;21:540-1.

[46] Roche AJ, Calder JD. Achilles tendinopathy: a review of the current concepts of treatment. Bone Joint J 2013;95:1299-307.

[47] Lundblad RL, Bradshaw RA, Gabriel D, et al. A review of the therapeutic uses of thrombin. Thromb Haemost 2004; 91:851-60.

[48] Knobloch K. Tendinopathy and drugs-potential implications for beneficial and detrimental effects on painful tendons. J Sci Med Sport 2009;12:423.

[49] Virchenko O, Aspenberg P, Lindahl TL. Low molecular weight heparin impairs tendon repair. J Bone Joint Surg $\mathrm{Br}$ 2008;90:388-92.

[50] Tanne JH. FDA adds "black box" warning to fuoroquinolone antibiotics. BMJ 2008, http://dx.doi.org/10.1136/bmj.a816.

[51] Kaleagasioglu F, Olcay E. Fluoroquinolone-induced tendinopathy: etiology and preventive measures. Tohoku J Exp Med 2012;226:251-8.

[52] Gotoh M, Mitsui $Y$, Shibata $H$, et al. Increased matrix metalloprotease-3 gene expression in ruptured rotator cuff tendons is associated with postoperative tendon retear. Knee Surg Sports Traumatol Arthrosc 2013;12:1807-12.

[53] Pasternak B, Fellenius M, Aspenberg P. Doxycycline impairs tendon repair in rats. Acta Orthop Belg 2006;72:756-60.

[54] Pasternak B, Missios A, Askendal A, et al. Doxycycline-coated sutures improve the suture-holding capacity of the rat Achilles tendon. Acta Orthop 2007;78:680-6.

[55] Bedi A, Fox AJ, Kovacevic D, et al. Doxycycline-mediated inhibition of matrix metalloproteinases improves healing after rotator cuff repair. Am J Sports Med 2010;38:308-17.

[56] Kessler MW, Barr J, Greenwald R, et al. Enhancement of Achilles tendon repair mediated by matrix metalloproteinase inhibition via systemic administration of doxycycline. J Orthop Res 2014;32:500-6.

[57] Marie I, Noblet C. Tendinopathies induites par les statines. John Libbey Euronext 2010;16:238-43.

[58] Kamio K, Liu XD, Sugiura H, et al. Statins inhibit matrix metalloproteinase release from human lung fibroblasts. Eur Respir J 2010;35:637-46.

[59] Marie I, Delafenêtre H, Massy N, et al. Complications tendineuses des statines : à propos de 96 observations. J Mal Vasc 2008;33:34-5.

[60] Kaleagasioglu F, Olcay E, Olgac V. Statin-induced calcific Achilles tendinopathy in rats: comparison of biomechanical and histopathological effects of simvastatin, atorvastatin and rosuvastatin. Knee Surg Sports Traumatol Arthrosc 2015;25:1884-91.

[61] Davis ME, Korn MA, Gumucio JP, et al. Simvastatin reduces fibrosis and protects against muscle weakness after massive rotator cuff tear. J Shoulder Elbow Surg 2015;24:280-7.

[62] Knobloch K. Drug-Induced Tendon Disorders. In: Ackermann P, Hart D, editors. Metabolic Influences on Risk for Tendon Disorders. Advances in Experimental Medicine and Biology. Springer; 2016. p. 229-38.

[63] Marsolais D, Frenette J. Inflammation et réparation tendineuse. Med Sci 2005;21:181-6.

[64] Dahners LE, Gilbert JA, Lester GE, et al. The effect of a nonsteroidal antiinflammatory drug on the healing of ligaments. Am J Sports Med 1988;16:641-6.

[65] Elder CL, Dahners LE, Weinhold PS. A cyclooxygenase-2 inhibitor impairs ligament healing in the rat. Am J Sports Med 2001;29:801-5.

[66] Marsolais D, Côté CH, Frenette J. Nonsteroidal antiinflammatory drug reduces neutrophil and macrophage accumulation but does not improve tendon regeneration. Lab Invest 2003;83:991-9.

[67] Lapointe BM, Frément $P$, Côté $\mathrm{CH}$. Adaptation to lengthening contractions is independent of voluntary muscle recruitment but relies on inflammation. Am J Physiol 2002;282: $323-9$.

[68] Galatz LM, Silva MJ, Rothermich SY, et al. Nicotine delays tendon-to-bone healing in a rat shoulder model. J Bone Joint Surg Am 2006;88:2027-34.

[69] Baumgarten KM, Gerlach D, Galatz LM, et al. Cigarette smoking increases the risk for rotator cuff tears. Clin Orthop Relat Res 2010;468:1534-41.

[70] Carbon S, Gumina S, Arceri V, et al. The impact of preoperative smoking habit on rotator cuff tear: cigarette smoking influences rotator cuff tear sizes. J Shoulder Elbow Surg 2012;21:56-60.

[71] Titchener AG, White JJ, Hinchliffe, et al. Comorbidities in rotator cuff disease: a case-control study. J Shoulder Elbow Surg 2014;23:1282-8.

[72] Godic A, Poljšak B, Adamic $M$, et al. The role of antioxidants in skin cancer prevention and treatment. Oxid Med Cell Longev 2014, http://dx.doi.org/10.1155/2014/860479.

[73] Kannus P, Paavola M, Józsa L. Aging and degeneration of tendons. In: Maffulli N, Renström P, Leadbetter WB, editors. Tendon Injuries. London: Springer; 2015. p. 25-31.

[74] Galera O, Gleizes-Cervera S, Pillard F, et al. Prevalence of injuries in triathletes from a French league. Apunts Med Esport 2012;47:9-15.

[75] Fusini F, Bisicchia S, Bottegoni, et al. Nutraceutical supplement in the management of tendinopathies: a systematic review. Muscles Ligaments Tendons J 2016;6:48-57.

[76] Poulsen RC, Zarei A, Sabokbar A, et al. Tendon, a vitamin Dresponsive tissue - why the British weather may not just be bad for your bones! Int J Exp Pathol 2013;94:20.

[77] Buhrmann C, Mobasheri A, Busch F, et al. Curcumin modulates nuclear factor kappaB (NF-kappaB)-mediated inflammation in 
human tenocytes in vitro: role of the phosphatidylinositol 3kinase/Akt pathway. J Biol Chem 2011;286:28556-66.

[78] Subhashini, Chauhan PS, Kumari S, et al. Intranasal curcumin and its evaluation in murine model of asthma. Int Immunopharmacol 2013;17:733-43.

[79] Fredberg U, Bolvig L. Significance of ultrasonographically detected asymptomatic tendinosis in the patellar and achilles tendons of elite soccer players: a longitudinal study. Am J Sports Med 2002;30:488-91.

[80] Fredberg U, Bolvig L, Andersen N. Prophylactic training in asymptomatic soccer players with ultrasonographic abnormalities in Achilles and patellar tendons. Am J Sports Med 2008;36:451-60.

[81] Cook J, Plazsnik R, Kiss ZS, Malliars P, Morris ME, Luca JD. High reproducibility of patellar tendon vascularity assessed by colour Doppler ultrasonography: a reliable measurement tool for quantifying tendon pathology. $\mathrm{Br} \mathrm{J}$ Sports Med 2005;39:700-3.

[82] Gisslen K, Alfredson H. Neovascularisation and pain in jumper's knee: a prospective clinical and sonographic study in elite volleyball players. Br J Sports Med 2005;37:423-8.
[83] Turk Z, Misur I, Turk N, et al. Rat tissue collagen modified by advanced glycation: correlation with duration of diabetes and glycemic control. Clin Chem Lab Med 1999;37:813-20.

[84] Perez-Ruiz F, Dalbeth N, Bardin T. A review of uric acid, crystal deposition disease, and gout. Adv Ther 2015;32: $31-41$.

[85] Lowes DA, Wallace C, Murphy MP, et al. The mitochondria targeted antioxidant MitoQ protects against fluoroquinoloneinduced oxidative stress and mitochondrial membrane damage in human Achilles tendon cells. Free Radic Res 2009;43:323-8.

[86] Jauslin ML, Meier T, Smith RAJ, Murphy MP. Mitochondria targeted antioxidants protect Friedreich ataxia fibroblasts from endogenous oxidative stress more effectively than untargeted antioxidants. FASEB J 2003;17:1972-4.

[87] Nagai R, Murray DB, Metz TO, et al. Chelation: a fundamental mechanism of action of AGE inhibitors, AGE breakers, and other inhibitors of diabetes complications. Diabetes 2012;61:549-59. 